\title{
Reverberation nulling and echo enhancement at low frequency using waveguide invariance
}

\author{
GUO GuoQiang*, YANG YiXin \& SUN Chao \\ Institute of Acoustic Engineering, Northwestern Polytechnical University, Xi'an 710072, China
}

Received April 25, 2010; accepted July 6, 2010

Based on the fact that the transfer function vector between a source receiver array and the dominant scatterer of boundary reverberation at a range can be obtained from the corresponding reverberations scattered from this range cell, a reverberation nulling concept using time reversal processing has been proposed. However, current reverberation nulling methods have certain limitations when applied into practice, which would null boundary reverberation and target echo simultaneously. As a solution, a passive reverberation nulling and echo enhancement method at low frequency using waveguide invariance is proposed in this paper. In this method, the reverberation subspace for the target range cell is not obtained directly from the return signals scattered by the target range cell but from the return signals scattered by a range cell located before the target using waveguide invariance, so as to suppress the reverberation embodied in the target echo by passive reverberation nulling. Besides, a range-dependent optimal weighting vector rather than conventional projector matrix is deduced to null the reverberation component meanwhile maximizing the target echo, thereby enhancing the echo-to-reverberation ratio furthest. Numerical simulations in typical range-independent shallow water environment demonstrate the efficacy and the improved performance of the proposed method for echo-to-reverberation enhancement.

reverberation nulling, echo-to-reverberation enhancement, waveguide invariance, time reversal

Citation: Guo G Q, Yang Y X, Sun C. Reverberation nulling and echo enhancement at low frequency using waveguide invariance. Chinese Sci Bull, 2011, 56: 142-150, doi: 10.1007/s11434-010-4207-z

In shallow water, the performance of active sonar systems is severely degraded by bottom reverberation that is produced by the sonar itself and determined by the characteristics of transmission channel. Thus, the suppression of reverberation is highly desirable. Time reversal (TR) [1-6] processing utilizes the reciprocity of wave transmission in static medium and focuses acoustic energy adaptively at the origin of the probe signal without prior knowledge of the medium and the transducer array. Recently, this new adaptive focusing method has been applied to echo enhancement and reverberation suppression in shallow water [7-10].

Assuming that a probe source near the target was available, Kim et al. [7] studied the echo-to-reverberation enhancement of time reversal focusing using a time reversal

*Corresponding author (email: guoguo3303@mail.nwpu.edu.cn) mirror (TRM) directly. Consequent echo-to-reverberation enhancement was demonstrated experimentally in a shallow water environment. However, a major drawback of the TR processing for practical use in echo-to-reverberation enhancement is the requirement of a cooperating probe source near the target.

Under the condition that no cooperating probe source near the target was available, Song et al. [8-10] proposed a reverberation nulling concept using TRM. This reverberation nulling concept is based on the fact that the transfer function vector between a source receiver array (SRA or TRM) and the dominant scatterers of boundary reverberation can be obtained from the corresponding reverberation returns scattered from this range cell. Song et al. [8,9] initially investigated the active reverberation nulling using a TRM, which could null the acoustic energy incident on the 
corresponding scattering interface by applying an excitation weighting vector that is in the complementary subspace orthogonal to the focusing vector on the SRA, so as to null the corresponding bottom reverberation returns from this range cell. Apparently, this active reverberation nulling requires an active transmission from the SRA to the waveguide to minimize the sound energy interacting with the boundaries at a given range. Because of the limited ranges over which the boundary interaction of the sound energy is suppressed, different excitation vectors must be transmitted for each range cell of interest for target detection. As an alternative, Song et al. [10] then proposed a passive processing method for reverberation nulling, which is called a passive reverberation nulling method in this paper. In a similar fashion, the transfer function vectors between the SRA and the dominant scatterers of boundary reverberation at each range cell are extracted from the observed reverberation returns. And then the obtained transfer function vectors are employed in subsequent derivation of a rangedependent sequence of projection operators, which are later applied to the raw received data vectors to suppress the reverberation component embedded in return signals without specialized active retransmissions for each range cell.

Unfortunately, both active and passive reverberation nulling methods suffered from certain limitations when applied to practice. The basis of these two methods is that a time-windowed segment of reverberation enables estimation of the transfer function vector between SRA and the corresponding range cell along the bottom [11]. In previous studies, only boundary reverberation that returns from the conventional active transmissions were considered and target-free reverberation observations were assumed to be available to construct a reverberation subspace and a weighting vector of SRA for retransmission (or a projection matrix for passive reverberation nulling). If a target exists in the waveguide, however, the return signals from the target range contain not only the boundary reverberation but also the target echo, which are difficult to separate to obtain target-free reverberation observations without any prior knowledge. Thus, the reverberation subspace constructed from these "mixed" return signals would inherently contain the response information between the source receiver array elements and the target. Consequently, the reverberation nulling using this reverberation subspace would reduce the bottom reverberation and the target echo simultaneously.

To solve the above problem, an echo-to-reverberation enhancement method based on decomposition of the time reversal operator (DORT) [12-16] with forward and backward reverberation nulling has been proposed [17]. This method can focus sound energy at the target while nulling the energy at the bottom near the target range simultaneously, substantially enhancing the echo-to-reverberation ratio without a probe source and prior knowledge about the relative scattering intensity of target and bottom. Nevertheless, it is an active processing method and requires transmit- ting different nulling excitation vectors for each range cell of interest for target detection, which has heavy computation load.

In terms of passive processing, a passive reverberation nulling and echo enhancement method using waveguide invariance is proposed in this paper. A major advance is that the reverberation subspace for the target range cell is obtained from the return signals scattered by a range cell located before the target using waveguide invariance, rather than directly from the return signals scattered by the target range cell. Therefore, reverberation is significantly suppressed while the target echo is maintained. Moreover, a range-dependent optimal weighting vector is deduced rather than a conventional projector matrix to null the reverberation component in the return signals while maximizing the target echo simultaneously, therefore enhancing the echo-toreverberation ratio furthest. Since passive processing is used, this method eliminates the complexity of transmitting different nulling excitation vectors for each range of interest, which is required in the active time reversal reverberation nulling. And since the waveguide invariance is used to predict the reverberation subspace, this method also remedies the shortage of current passive reverberation nulling, which may diminish the target echo and bottom reverberation simultaneously.

\section{Reverberation nulling}

The basic theory of TR reverberation nulling was described in detail in [8]. A brief review is given in this section.

An SAR consisting of $N$ elements is configured to transmit a pulse of length $\tau$ to the waveguide starting at $t=t_{0}$, and then the resulting reverberation time series are recorded on each element. Assuming that only single scattering is important, the scattering from a specific range $R$ will contribute to the received reverberation over a time window:

$$
w(t, R, \Delta)= \begin{cases}1, & \left|t-t_{c}-t_{0}\right| \leqslant \Delta / 2, \\ 0, & \left|t-t_{c}-t_{0}\right|>\Delta / 2,\end{cases}
$$

where $t_{c}=2 R / c_{0}$ is the approximate round-trip travel time, $c_{0}$ is the average modal group speed, and $\Delta$ is the duration width of the temporal window, which depends on the source pulse length and the dispersive properties of the waveguide. For a narrowband signal, it is typical to choose $\Delta=\tau$. Due to the dispersion in the waveguide, the reverberation in the widowed time series is mainly contributed by a range cell (a span of ranges of width $\left.\Delta R=c_{0} \Delta / 2=c_{0} \tau / 2\right)$. Thus, a timegated portion of the reverberation can be reduced to a single (extended) source problem for a specific range cell. The scattering model between SRA and the bottom interface around the range $R$ is presented in Figure 1. The horizontal distance between SRA and the target $S_{1}$ (or the bottom scatter $\mathrm{S}_{2}$ ) is $R$, and the bottom sactterers $\mathrm{S}_{3}, \mathrm{~S}_{2}$ and $\mathrm{S}_{4}$ are 


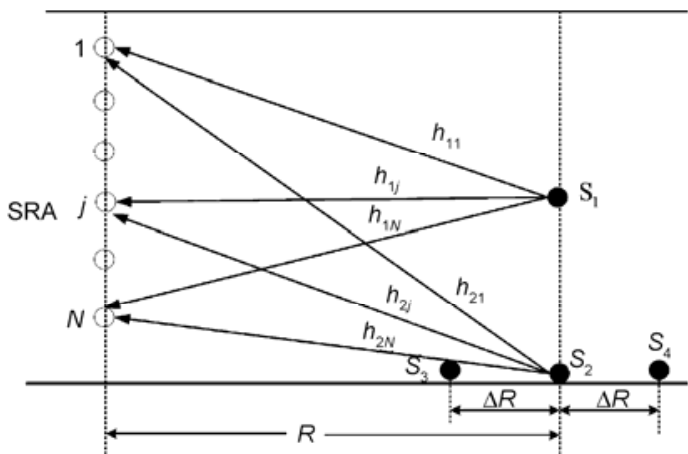

Figure 1 The scattering model from the target and bottom scatterers to SRA.

neighboring with horizontal interval $\Delta R=c_{0} \tau / 2$. The transmission function vector between the target $S_{1}$ and SRA is denoted by $\boldsymbol{h}_{1}(\omega)=\left[h_{11}, h_{12}, \cdots, h_{1 N}\right]^{\mathrm{T}}$. Similarly, the transmission function vectors between the bottom scatterers $S_{2}, S_{3}$ and $\mathrm{S}_{4}$ and SRA are denoted by $\boldsymbol{h}_{2}(\omega), \boldsymbol{h}_{3}(\omega)$ and $\boldsymbol{h}_{4}(\omega)$, respectively.

For a given range of interest, a time reversal operator (TRO) $[11,18,19]$ can be constructed from the return signals received by SRA as

$$
\mathrm{TRO} \equiv \boldsymbol{K}^{*}(\omega) \boldsymbol{K}(\omega)=\left(\tilde{\boldsymbol{K}}(\omega) \tilde{\boldsymbol{K}}^{\mathrm{H}}(\omega)\right)^{*}=\boldsymbol{R}^{*}(\omega),
$$

where the superscript "**" denotes phase conjugation, $\mathrm{H}$ represents Hermitian transpose, $\omega$ is the frequency, $\boldsymbol{K}(\omega)$ is transfer matrix, and $\tilde{\boldsymbol{K}}(\omega)$ is the return signal matrix which can be written as

$$
\tilde{\boldsymbol{K}}(\omega)=\boldsymbol{K}(\omega) \boldsymbol{E}(\omega),
$$

where $\boldsymbol{E}=\left[\boldsymbol{e}_{1}, \boldsymbol{e}_{2}, \cdots, \boldsymbol{e}_{N}\right]$ is an excitation matrix whose column vectors $\boldsymbol{e}_{i}$ represent $N$ forms of excitation of the array (beams), and it satisfies the orthogonality, i.e. $\boldsymbol{E} \boldsymbol{E}^{\mathrm{H}}=\boldsymbol{E}^{\mathrm{H}} \boldsymbol{E}=\boldsymbol{I}$, where $\boldsymbol{I}$ is an identity matrix with size $N$. Then eigendecomposition of the TRO yields

$$
\boldsymbol{R}(\omega)=\boldsymbol{U} \Lambda \boldsymbol{U}^{\mathrm{H}}+\boldsymbol{V} \Omega \boldsymbol{V}^{\mathrm{H}},
$$

where $\Lambda$ is a diagonal matrix containing $K$ largest eigenvalues, $\boldsymbol{U}$ is an $N \times K$ matrix of the corresponding eigenvectors $\left(\boldsymbol{u}_{i}, i=1, \ldots K\right)$ spanning a $K$-dimensional reverberation subspace, $\Omega$ is a diagonal matrix containing $N-K$ smallest eigenvalues, and $\boldsymbol{V}$ is an $N \times(N-K)$ matrix of the corresponding eigenvectors $\left(v_{i}, i=1, \cdots, N-K\right)$ spanning a $(N-K)$-dimensional noise subspace.

Ideally if there is no target at this range, TRO is a rank-1 matrix, a single reverberation return (a single snapshot) allows construction of the TRO. In this case, the signal subspace is 1 -dimensional (i.e. $K=1$ ) and can be represented by a single eigenvector $\boldsymbol{u}_{1}$, which is proportional to the transfer function vector between the source receiver array elements and the dominant scatterers of boundary reverberation. Using this eigenvector as an excitation weighting vector of
SRA for retransmission, a strong return would be yielded in the resulting reverberation at the corresponding time window [11]. For active reverberation nulling case, if an arbitrary vector in the $(N-1)$-dimensional noise subspace, which is orthogonal to the signal subspace, is chosen as an excitation weighting vector of SRA for retransmission, the sound energy interacting with the range cell along the bottom will be reduced and the resulting reverberation will yield a null at the corresponding time window [8,9]. For passive reverberation nulling case, however, instead of retransmitting sound energy to the waveguide using nulling excitation weighting vectors, a projection operator is directly applied to the received signal data vectors produced by conventional transmission. The projection operator can be constructed in terms of an $N \times N$ projection matrix which is orthogonal to the reverberation subspace, so that

$$
\boldsymbol{P}=\boldsymbol{I}-\boldsymbol{U} \boldsymbol{U}^{\mathrm{H}}=\boldsymbol{I}-\sum_{i=1}^{K} \boldsymbol{u}_{i} \boldsymbol{u}_{i}^{\mathrm{H}} .
$$

Consequently, passive reverberation nulling can be achieved by applying this projector matrix $\boldsymbol{P}$ directly to the reverberation returns [10].

If there is a target in the waveguide, however, the return signals from the target range contain both the boundary reverberation and the target echo. Then the signal subspace obtained by DORT from these return signals is 2-dimensional (i.e. $K=2$ ) with eigenvector $\boldsymbol{u}_{1}$ and $\boldsymbol{u}_{2}$, which reflect the information in reverberation subspace and the target echo subspace, respectively. Without prior knowledge about the relative scattering intensity of target and bottom scatterers, it is impossible to extract a pure reverberation subspace for reverberation nulling. Therefore, both the active and passive reverberation nulling methods would reduce the bottom reverberation and the target echo simultaneously $[10,17]$.

One solution using the active processing is the echo-toreverberation enhancement method based on DORT with forward and backward reverberation nulling, as proposed in [17]. Without a probe source or knowledge of the relative scattering intensity of target and bottom, this method enables sound energy focus at the target while simultaneously suppress the energy at the bottom near the target range, thereby enhancing the echo-to-reverberation ratio. Another solution in terms of passive processing for echo-to-reverberation ratio enhancement will be described in detail in next section.

\section{Echo-to-reverberation enhancement}

\subsection{Waveguide invariance}

Waveguide invariance illustrates that low frequency broadband acoustic pressure spectrum in an oceanic waveguide has stable frequency-range interference pattern [20,21]. And based on this, Zhang et al. [22] demonstrated a linear phase 
relationship between the frequency response functions of two points with certain longitudinal distance. Recently, the waveguide invariance is used to improve the longitudinal correlations of acoustical field [22] and to shift the range of time reversal focusing [23,24]. Here, the waveguide invariance is employed to predict the reverberation subspace of the target range cell from the return signals scattered by a range cell before the target.

Assume that a low frequency acoustic signal is transmitted into a range-independent waveguide environment. Let $G\left(R, z_{r}, z_{s}, \omega\right)$ denote the response function of the ocean channel, where the distance between the source and the receiver is $R$ and the depths of the source and receiver are $z_{s}$ and $z_{r}$ respectively. If $\Delta R<<R$, it has been proved that [21]

$$
\left|G\left(R+\Delta R, z_{r}, z_{s}, \omega+\Delta \omega\right)\right|^{2} \approx\left|G\left(R, z_{r}, z_{s}, \omega\right)\right|^{2},
$$

and the frequency shift $\Delta \omega$ and range shift $\Delta R$ satisfy

$$
\frac{\Delta R}{R}=\frac{1}{\beta} \frac{\Delta \omega}{\omega},
$$

where $\beta$ is the waveguide invariant introduced by Chuprov et al. [20] and is denoted by

$$
\beta \equiv \frac{R}{\omega} \frac{\mathrm{d} \omega}{\mathrm{d} R}=-\frac{\mathrm{d}\left(1 / v^{p}\right)}{\mathrm{d}\left(1 / v^{g}\right)},
$$

where $\vartheta^{p}$ and $v^{g}$ are the averaged phase velocity and group velocity, respectively.

According to the normal mode theory, a linear phase relationship between $G\left(R, z_{r}, z_{s}, \omega\right)$ and $G\left(R+\Delta R, z_{r}, z_{s}, \omega+\Delta \omega\right)$ has been proved as [22]

$$
G\left(R+\Delta R, z_{r}, z_{s}, \omega+\Delta \omega\right) \approx \exp (i \omega \tau(\Delta R)) \cdot G\left(R, z_{r}, z_{s}, \omega\right),(9)
$$

where the frequency shift $\Delta \omega$ and range shift $\Delta r$ still hold eq. (7). It was indicated that $\tau$ varies insignificantly with frequency $\omega$, and can be regarded as a function of $\Delta r$ only [22]. Thus, a linear phase relationship between the transfer function vectors $\boldsymbol{h}_{2}(\omega)$ and $\boldsymbol{h}_{3}(\omega)$, denoted in Figure 1, can be given by

$$
\boldsymbol{h}_{3}(\omega-\Delta \omega) \approx \exp (-i \omega \tau(\Delta R)) \boldsymbol{h}_{2}(\omega) .
$$

\subsection{Passive reverberation nulling using waveguide invariance}

According to the scattering model shown in Figure 1, the return signals scattered from the target range $R$ and received by SRA contain both the boundary reverberation and the target echo, which can be written in frequency domain by

$$
\boldsymbol{x}(R, \omega)=\boldsymbol{s}(\omega)+\boldsymbol{r}(R, \omega)+\boldsymbol{n}(\omega),
$$

where $s(\omega)$ is the target echo, $\boldsymbol{r}(R, \omega)$ is the reverberation scattered by the bottom scatterer at the target range $R$ (i.e. $\mathrm{S}_{2}$ ), and $\boldsymbol{n}(\omega)$ is the environment noise, which can be regarded as an additive white noise and is uncorrelated with target echo as well as reverberation. Using these return signals and DORT as referred in Section 1, a signal subspace $\boldsymbol{U}(R, \omega)$ can be determined, which includes both echo and reverberation subspaces with eigenvectors $\boldsymbol{u}_{i}(R, \omega)(i=1, \cdots$, $K)$. Since no prior knowledge about the relative scattering intensity of the target and the bottom scatterer is available, it is impossible to distinguish which corresponds to the target echo and which corresponds to the reverberation. Thus, it is impossible to extract a pure reverberation subspace from the whole signal subspace for reverberation nulling.

In fact, the return signals received by SRA at the moment before the target echo appearing are dominated by the reverberation produced by bottom scatterer $\mathrm{S}_{3}$, which can be written as

$$
\boldsymbol{x}(R-\Delta R, \omega)=\boldsymbol{r}(R-\Delta R, \omega)+\boldsymbol{n}(\omega) .
$$

From these "earlier" return signals, a pure reverberation subspace $\boldsymbol{U}(R-\Delta R, \omega)$, associated with eigenvectors $\boldsymbol{u}_{i}(R-$ $\Delta R, \omega)\left(i=1, \ldots K^{\prime}\right)$, can be obtained by DORT. Ideally, the rank of reverberation subspace will be equal to 1 (i.e. $K=1$ ), and the eigenvector $\boldsymbol{u}_{1}$ is proportional to the transfer function vector between the SRA and the boundary scatterer $\mathrm{S}_{3}$, i.e. $\boldsymbol{u}_{1}(R-\Delta R, \omega) \approx \boldsymbol{h}_{3}(\omega)$.

If the duration of the signal emitted by SRA is short (i.e. $\tau$ is small) and the distance between the bottom sactterers $S_{2}$ and $\mathrm{S}_{3}$ is small (i.e. $\Delta R=c \tau / 2$ is small), then the reverberations from these two range cells can be assumed to be approximately stationary and highly correlated [25]. Intuitively, the reverberation subspace $U(R-\Delta R, \omega)$ can be directly used as a predictor (or estimate) for the reverberation subspace at the target range (i.e. $\hat{\boldsymbol{U}}(R, \omega)=\boldsymbol{U}(R-\Delta R, \omega))$. Then the projection matrix $\hat{\boldsymbol{P}}$ in eq. (5) is constructed as

$$
\begin{aligned}
\hat{\boldsymbol{P}} & =\boldsymbol{I}-\sum_{i=1}^{K} \hat{\boldsymbol{u}}_{i}(R, \omega) \hat{\boldsymbol{u}}_{i}^{\mathrm{H}}(R, \omega) \\
& =\boldsymbol{I}-\sum_{i=1}^{K} \boldsymbol{u}_{i}(R-\Delta R, \omega) \boldsymbol{u}_{i}^{\mathrm{H}}(R-\Delta R, \omega) .
\end{aligned}
$$

Simulations which will be presented in Section 3 indicate that passive reverberation nulling using this projection matrix will reduce the reverberation embedded in the target echo to lower levels if $\Delta R$ (or $\tau$ ) is small. As $\Delta R$ (or $\tau$ ) increases, however, the difference between the predicted reverberation subspace and the real one will become larger, and then the performance of the reverberation nulling will degrade. Herein, the waveguide invariance is used to solve this problem. According to eq. (10), the eigenvector is approximated as

$$
\boldsymbol{u}_{1}(R-\Delta R, \omega-\Delta \omega) \approx \boldsymbol{h}_{3}(\omega-\Delta \omega) \approx \exp (-i \omega \tau) \boldsymbol{h}_{3}(\omega) .
$$

Thus, a pure and exact reverberation subspace $\boldsymbol{u}_{1}(R, \omega)$ for the target range $R$ can be determined by DORT from the reverberations scattered by the boundary interface at range 
$R-\Delta R$ with frequency shift $\Delta \omega$, although there is a phase difference $\exp (-i \omega \tau)$ between the eigenvector $\boldsymbol{u}_{1}(R-\Delta R, \omega$ $\Delta \omega)$ and the transfer function vector $\boldsymbol{h}_{2}(\omega)$. Using this reverberation subspace as a forward prediction of the reverberation subspace for target range (i.e. $\hat{\boldsymbol{u}}_{i}(R, \omega)=\boldsymbol{u}_{i}(R-\Delta R, \omega-$ $\Delta \omega)$ ), the corresponding projection matrix $\hat{\boldsymbol{P}}$ in eq. (5) is

$$
\begin{aligned}
\hat{\boldsymbol{P}} & =\boldsymbol{I}-\sum_{i=1}^{K} \hat{\boldsymbol{u}}_{i}(R, \omega) \hat{\boldsymbol{u}}_{i}^{\mathrm{H}}(R, \omega) \\
& =\boldsymbol{I}-\sum_{i=1}^{K} \boldsymbol{u}_{i}(R-\Delta R, \omega-\Delta \omega) \boldsymbol{u}_{i}^{\mathrm{H}}(R-\Delta R, \omega-\Delta \omega) .
\end{aligned}
$$

Once derived projection matrix $\hat{\boldsymbol{P}}$ is applied to return signals (i.e. $\hat{\boldsymbol{P}} \boldsymbol{x}(R, \omega)=\hat{\boldsymbol{P}} \boldsymbol{s}(\omega)+\hat{\boldsymbol{P}} \boldsymbol{r}(R, \omega))$, the reverberation component will be expectedly suppressed so that the echo-to-reverberation ratio is enhanced (i.e. $|\hat{\boldsymbol{P}} \boldsymbol{r}(R, \omega)|$ $<<|\hat{\boldsymbol{P}} \boldsymbol{s}(\omega)|)$.

Since priori knowledge of target range is usually unavailable in real application, the proposed method can be realized by sliding (moving) a window as follows. Firstly, the return signals with a bandwidth received by SRA are divided into segments, each of which is transformed into the frequency domain by fast Fourier transform. Then, a reverberation subspace, denoted by $\boldsymbol{U}\left(R_{k}, \omega-\Delta \omega\right)$, is obtained from the $k$ th segment data with a certain frequency shift to construct a project matrix $\hat{\boldsymbol{P}}$ in eq. (15). After that, the projection matrix $\hat{\boldsymbol{P}}$ is applied to the next segment of the return signal data $\boldsymbol{x}\left(R_{k+1}, \omega\right)$ to null the reverberation component embedded in the $(k+1)$ th segment (i.e. $\left.\left|\operatorname{Pr}\left(R_{k+1}, \omega\right)\right| \approx 0\right)$. Finally, the whole reverberation component embedded in the return signals can be suppressed by the above processing for each segment truncated by the sliding (moving) window as $k$ increase, ultimately improving the target detectability.

\subsection{Target echo enhancement}

The passive reverberation nulling described in previous section mainly focused on the reverberation nulling and little consideration was given to echo enhancement. Since the signal subspace $\boldsymbol{U}(R, \omega)$ contains the transfer function vector information between the target and SRA, it is possible to further enhance the target echo using this signal subspace information. The basic strategy for the target echo enhancement is to find a weighting vector of SRA (denoted by $\boldsymbol{w}$ ) to null the reverberation component (i.e. $\boldsymbol{w}^{\mathrm{T}} \boldsymbol{r}(R, \omega)=0$ ), meanwhile maximizing the target echo strength (i.e. $\max \left|\boldsymbol{w}^{\mathrm{T}} \boldsymbol{s}(\omega)\right|^{2}$ ). In this way, a constrained optimization problem can be constructed as

$$
\begin{gathered}
\max _{w}(I(\boldsymbol{w}))=\max _{\boldsymbol{w}}\left(\boldsymbol{w}^{\mathrm{H}} \boldsymbol{s}^{*}(\omega) \boldsymbol{s}^{\mathrm{T}}(\omega) \boldsymbol{w}\right) \\
\text { s.t. } \quad \boldsymbol{w}^{\mathrm{T}} \boldsymbol{r}(R, \omega)=0, \quad \boldsymbol{w}^{\mathrm{H}} \boldsymbol{w}=1 .
\end{gathered}
$$

Because the echo information $s(\omega)$ and the reverberation information $\boldsymbol{r}(R, \omega)$ are not apparently available from the return signal $\boldsymbol{x}(\omega)$, the constrain of the reverberation nulling $\boldsymbol{w}^{\mathrm{T}} \boldsymbol{r}(R, \omega)=0$ is substituted by $\boldsymbol{w}^{\mathrm{T}} \boldsymbol{u}_{i}(R-\Delta R, \omega-\Delta \omega)=0, i=1, \cdots$, $K$, as in eq. (14), and the echo information $s(\omega)$ is substituted by $\boldsymbol{u}_{0}=\sum_{i=1}^{K} \boldsymbol{u}_{i}(R, \omega)$. According to the principle of DORT [12], it is known that $\boldsymbol{u}_{0}(R, \omega)$ is a linear combination of the transfer function vectors $\boldsymbol{h}_{1}(\omega)$ and $\boldsymbol{h}_{2}(\omega)$, i.e. $\boldsymbol{u}_{0}(R, \omega)=c_{1} \boldsymbol{h}_{1}(\omega)+c_{2} \boldsymbol{h}_{2}(\omega)$, where the coefficients $c_{1}$ and $c_{2}$ depend on the relative scattering intensity of the target and the bottom scatterer. Then, substituting $\boldsymbol{u}_{0}$ into $I(\boldsymbol{w})$ yields

$$
\begin{aligned}
I(\boldsymbol{w})= & \boldsymbol{w}^{\mathrm{H}} \boldsymbol{u}_{0}^{*} \boldsymbol{u}_{0}^{\mathrm{T}} \boldsymbol{w}=\boldsymbol{w}^{\mathrm{H}}\left(c_{1} \boldsymbol{h}_{1}(\omega)\right. \\
& \left.+c_{2} \boldsymbol{h}_{2}(\omega)\right)^{*}\left(c_{1} \boldsymbol{h}_{1}(\omega)+c_{2} \boldsymbol{h}_{2}(\omega)\right)^{\mathrm{T}} \boldsymbol{w} \\
= & \left|c_{1}\right|^{2} \boldsymbol{w}^{\mathrm{H}} \boldsymbol{h}_{1}^{*}(\omega) \boldsymbol{h}_{2}^{\mathrm{T}}(\omega) \boldsymbol{w}+c_{1}^{*} c_{2} \boldsymbol{w}^{\mathrm{H}} \boldsymbol{h}_{1}^{*}(\omega) \boldsymbol{h}_{2}^{\mathrm{T}}(\omega) \boldsymbol{w} \\
& +c_{1} c_{2}^{*} \boldsymbol{w}^{\mathrm{H}} \boldsymbol{h}_{2}^{*}(\omega) \boldsymbol{h}_{1}^{\mathrm{T}}(\omega) \boldsymbol{w}+\left|c_{2}\right|^{2} \boldsymbol{w}^{\mathrm{H}} \boldsymbol{h}_{2}^{*}(\omega) \boldsymbol{h}_{2}^{\mathrm{T}}(\omega) \boldsymbol{w} .
\end{aligned}
$$

Due to the bottom reverberation nulling constrains $\left(\boldsymbol{w}^{\mathrm{T}} \boldsymbol{u}_{i}(R-\Delta R, \omega-\Delta \omega)=0, i=1, \ldots K\right)$, the last three terms in eq. (17) are reasonably assumed to be zero, simplifying the problem to maximizing $I(w)$ is equivalent to maximizing the strength of target echo. Now, eq. (16) can be written as

$$
\begin{gathered}
\max _{\boldsymbol{w}}(I(\boldsymbol{w}))=\max _{\boldsymbol{w}}\left(\boldsymbol{w}^{\mathrm{H}} \boldsymbol{u}_{0}^{*} \boldsymbol{u}_{0}^{\mathrm{T}} \boldsymbol{w}\right) \\
\text { s.t. } \boldsymbol{w}^{\mathrm{T}} \hat{\boldsymbol{U}}=0, \quad \boldsymbol{w}^{\mathrm{H}} \boldsymbol{w}=1,
\end{gathered}
$$

where $\hat{\boldsymbol{U}}=\boldsymbol{U}(R-\Delta R, \omega-\Delta \omega)=\left[\boldsymbol{u}_{1}(R-\Delta R, \omega-\Delta \omega), \cdots, \boldsymbol{u}_{K}(R-\Delta R\right.$, $\omega-\Delta \omega)]$.

Using the projection matrix $\hat{\boldsymbol{P}}$ given in eq. (15), the solution vector of eq. (18) is

$$
\boldsymbol{w}=\hat{\boldsymbol{P}} \boldsymbol{u}=\left[\boldsymbol{I}-\hat{\boldsymbol{U}} \hat{\boldsymbol{U}}^{\mathrm{H}}\right] \boldsymbol{u},
$$

where $\boldsymbol{u}$ is a new $N$-dimension vector. Then eq. (18) can be reduced to

$$
\begin{gathered}
\max _{\boldsymbol{u}}(I(\boldsymbol{u}))=\max _{\boldsymbol{u}}\left(\boldsymbol{u}^{\mathrm{H}} \boldsymbol{P} \boldsymbol{u}_{0}^{*} \boldsymbol{u}_{0}^{\mathrm{T}} \boldsymbol{P} \boldsymbol{u}\right) \\
\text { s.t. } \boldsymbol{u}^{\mathrm{H}} \boldsymbol{P} \boldsymbol{u}=1 .
\end{gathered}
$$

Using Lagrange multiplier method to solve this constrained optimization problem, we can get

$$
\boldsymbol{u}_{\mathrm{opt}}=\frac{\boldsymbol{u}_{0}^{*}}{\sqrt{\boldsymbol{u}_{0}^{\mathrm{T}} \hat{\boldsymbol{P}} \boldsymbol{u}_{0}^{*}}} .
$$

Therefore, the final optimal solution of eq. (18) is given by

$$
\boldsymbol{w}_{\mathrm{opt}}=\hat{\boldsymbol{P}} \boldsymbol{u}_{\mathrm{opt}}=\frac{\hat{\boldsymbol{P}} \boldsymbol{u}_{0}^{*}}{\sqrt{\boldsymbol{u}_{0}^{\mathrm{T}} \hat{\boldsymbol{P}} \boldsymbol{u}_{0}^{*}}} .
$$

Like the projection matrix $\hat{\boldsymbol{P}}$ used in passive reverberation nulling, the optimal weight vector $\boldsymbol{w}_{\text {opt }}$ can be directly applied to the return signals (i.e. $\boldsymbol{w}_{\mathrm{opt}}^{\mathrm{T}} \boldsymbol{x}(R, \omega)=\boldsymbol{w}_{\mathrm{opt}}^{\mathrm{T}} \boldsymbol{s}(\omega)+$ 
$\left.\boldsymbol{w}_{\mathrm{opt}}^{\mathrm{T}} \boldsymbol{r}(R, \omega)\right)$ to null the reverberation component (i.e. $\left|\boldsymbol{w}_{\mathrm{opt}}^{\mathrm{T}} \boldsymbol{r}(R, \omega)\right| \approx 0$ ) while maximizing the target echo component simultaneously (i.e. $\max \left|\boldsymbol{w}_{\mathrm{opt}}^{\mathrm{T}} \boldsymbol{s}(\omega)\right|$ ), therefore, enhancing the echo-to-reverberation ratio further. As referred above, this method can be realized by a sliding (moving) window, so as to suppress the whole reverberation component embedded in the return signals while enhancing the target echo simultaneously if there does exist echo in the return signal data, therefore enhancing the echo-to-reverberation ratio furthest for target detection.

\section{Simulations}

In this section, the passive reverberation nulling and echo enhancement using waveguide invariance proposed in this paper is studied by numerical simulations in a range-independent waveguide environment. The simulation environment is similar to that of the experiment presented in [7], as shown in Figure 2. The SRA consists of 32 elements spanning the water column from $12 \mathrm{~m}$ to $105 \mathrm{~m}$ with $3 \mathrm{~m}$ inter-element spacing in the $120 \mathrm{~m}$ deep water. The soundspeed profile indicates a typical downward refracting environment. The target is at $60 \mathrm{~m}$ in depth and $4 \mathrm{~km}$ in range from the SRA (corresponding to $5.3 \mathrm{~s}$ round-trip travel time). And the scattering strength of the target is assumed to be equal to that of the bottom scatterers. The central frequency of the signal for simulations is $500 \mathrm{~Hz}$. Assume that the environment has no fluctuation during the processing. In this waveguide environment, $\beta \approx 1.2$, calculated in eq. (8). In real application, several local and global techniques can be used to extract the waveguide invariant from the spectrogram striations [26]. Only the bottom boundary scattering is considered. Shallow water normal mode reverberation model $[27,28]$ is used to calculate the reverberation returns from the bottom interface. And the acoustic field and normal modes are calculated by KRAKEN normal mode codes [29].

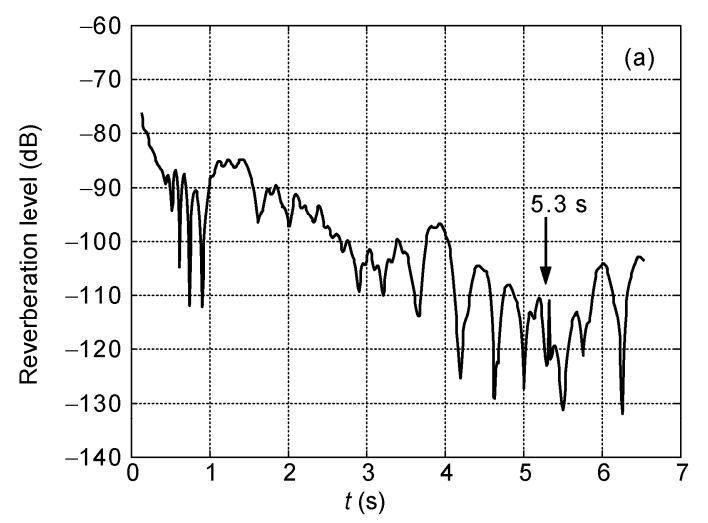

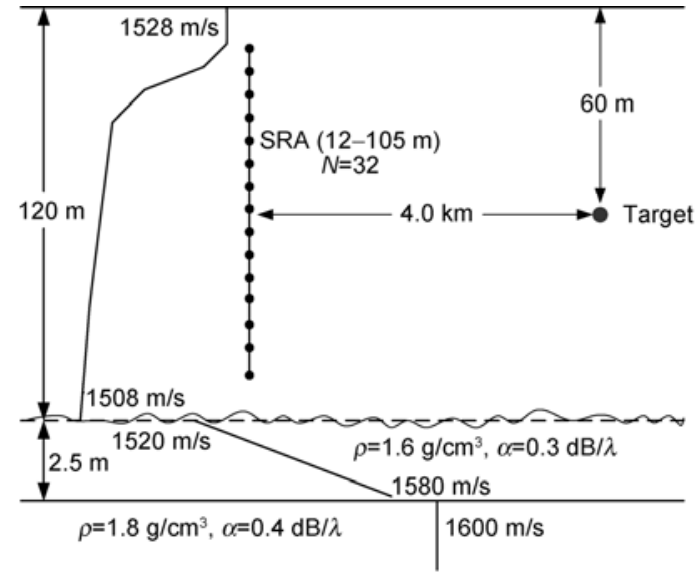

Figure 2 Shallow water environment used for numerical simulation.

Assuming that SRA ensonifies the waveguide by exciting all elements simultaneously (broadside transmission, BS), the bottom reverberations scattered from each range cell and the target echo are calculated separately using the normal model. And the returned signals are acquired by adding the simulated target echo to the bottom reverberations at around 5.3 s. Figure 3(a) shows the incoherent reverberation level received by SRA from $\mathrm{BS}$ transmission. It is observed that the echo at $5.3 \mathrm{~s}$ is submerged in bottom reverberation. Figure 3(b) shows the reverberation level received by SRA from the BS transmission after using conventional passive reverberation nulling discussed in Section 1. Because the reverberation subspace for the target range is directly obtained from the target echo together with reverberation returns from the target range, it contains both reverberation subspace and echo subspace information. Thus the reverberation and the echo are suppressed simultaneously by the passive reverberation nulling using the projector matrix $\boldsymbol{P}$ given in eq. (5).

In order to solve the above problem, a passive reverberation nulling method using waveguide invariance is proposed in this paper. As referred in Section 2.2, intuitively we trend

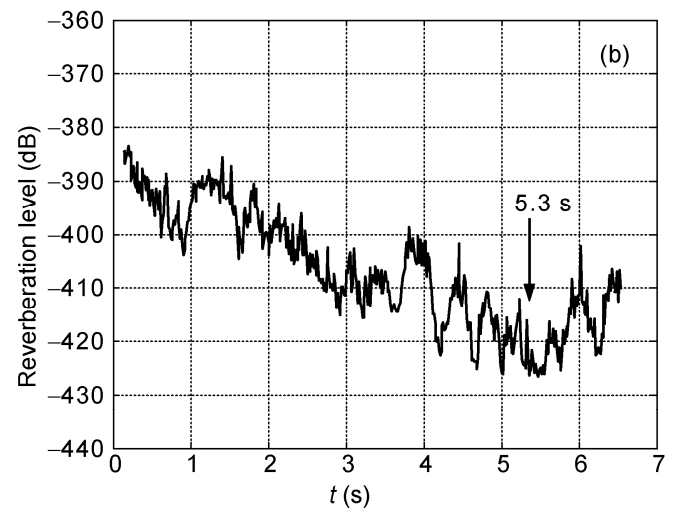

Figure 3 Reverberation levels from BS transmissions. (a) Incoherent reverberation level from the BS transmission; (b) the resulting reverberation level after passive reverberation nulling using the projector matrix given in eq. (5). 
to use the direct forward prediction, where the reverberation subspace $\boldsymbol{U}(R-\Delta R, \omega)$ is directly used as a predictor (or estimate) of the reverberation subspace for the target range (i.e. $\hat{\boldsymbol{U}}(R, \omega)=\boldsymbol{U}(R-\Delta R, \omega))$. Apparently, this direct forward prediction is not accurate enough. Integration of frequency shift into the forward prediction could improve its performance with appreciably enhanced correlation of the predicted reverberation subspace and the real one, as indicated in eq. (14).

Let $\Delta R=40 \mathrm{~m}$, which is determined by the signal duration $\tau$ as $\Delta R=c \tau / 2$. For passive reverberation nulling based on direct forward prediction, $\hat{\boldsymbol{U}}(R, \omega)=\boldsymbol{U}(R-\Delta R, \omega)$ is used to construct the projection matrix $\hat{\boldsymbol{P}}$ according to eq. (13). Figure 4(a) shows the resulting reverberation level after passive reverberation nulling using this projection matrix $\hat{\boldsymbol{P}}$. For passive reverberation nulling using waveguide invariance, $\hat{\boldsymbol{U}}(R, \omega)=\boldsymbol{U}(R-\Delta R, \omega-\Delta \omega)$ is used to construct the projection matrix $\hat{\boldsymbol{P}}$ according to eq. (15), where $\Delta \omega$ is calculated in eq. (7). The resulting reverberation level is presented in Figure 4(b). Similar results obtained by these two passive nulling methods when $\Delta R=80 \mathrm{~m}$ are shown in Figure 5. Comparing the reverberation nulling results given in Figures 4 and 5, it can be found that passive reverberation nulling using the projection matrix $\hat{\boldsymbol{P}}$ in eq. (15) can suppress the reverberation more efficiently and is relatively more robust as $\Delta R$ increases than that using the projection matrix $\hat{\boldsymbol{P}}$ in eq. (13).

As discussed in Section 2.3, an optimal weighting vector $\boldsymbol{w}_{\text {opt }}$ of SRA can be obtained from $\hat{\boldsymbol{P}}$ and $\boldsymbol{U}(R, \omega)$ to null the reverberation while promoting the target echo strength. For the case of passive reverberation nulling based on direct forward prediction, where $\hat{\boldsymbol{U}}(R, \omega)=\boldsymbol{U}(R-\Delta R, \omega)$ is used to construct the projection matrix $\hat{\boldsymbol{P}}$ according to eq. (13), the resulting reverberation level after reverberation nulling using the optimal weighting vector is shown in Figure 6(a). For the case of passive reverberation nulling using waveguide invariance, where $\hat{\boldsymbol{U}}(R, \omega)=\boldsymbol{U}(R-\Delta R, \omega)$ is used to construct the projection matrix $\hat{\boldsymbol{P}}$ according to eq. (15), the resulting reverberation level after reverberation nulling using the optimal weighting vector is shown in Figure 6(b). Comparing the reverberation nulling results given in Figures 6 and 5, it can be intuitively found that the echo levels after reverberation nulling by optimal weight vector and project matrix are approximately equal, while the reverberation levels after reverberation nulling by optimal weight vector
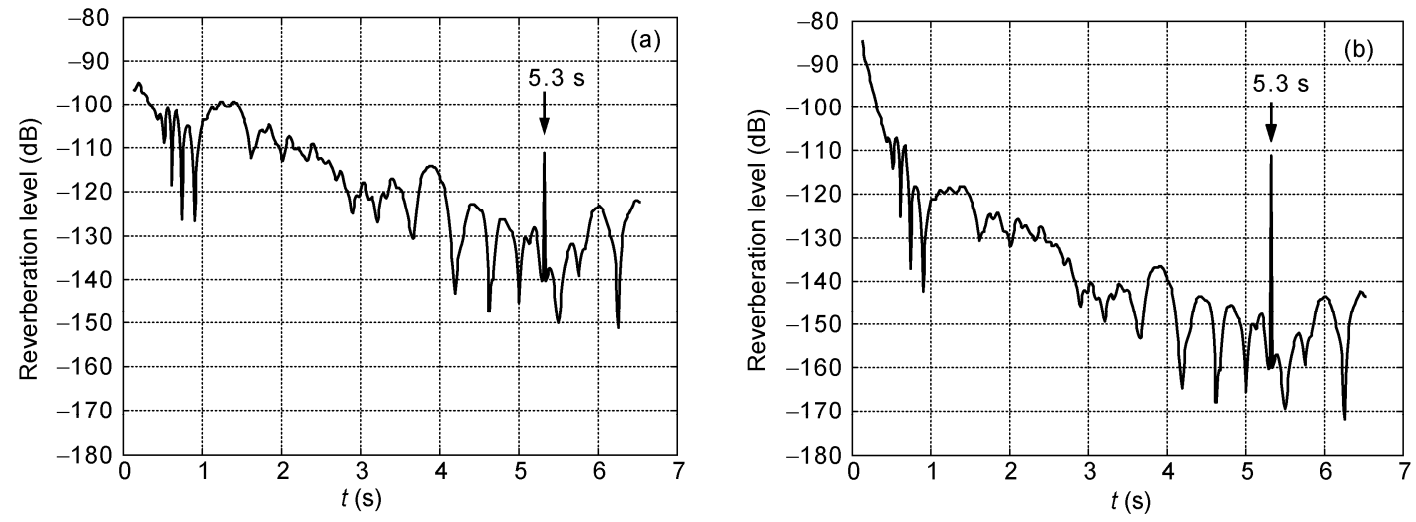

Figure 4 The resulting reverberation levels after passive reverberation nulling using the projection matrix given in eq. (13) (a), and passive reverberation nulling using the projection matrix given in eq. (15) (b), when $\Delta R=40 \mathrm{~m}$.
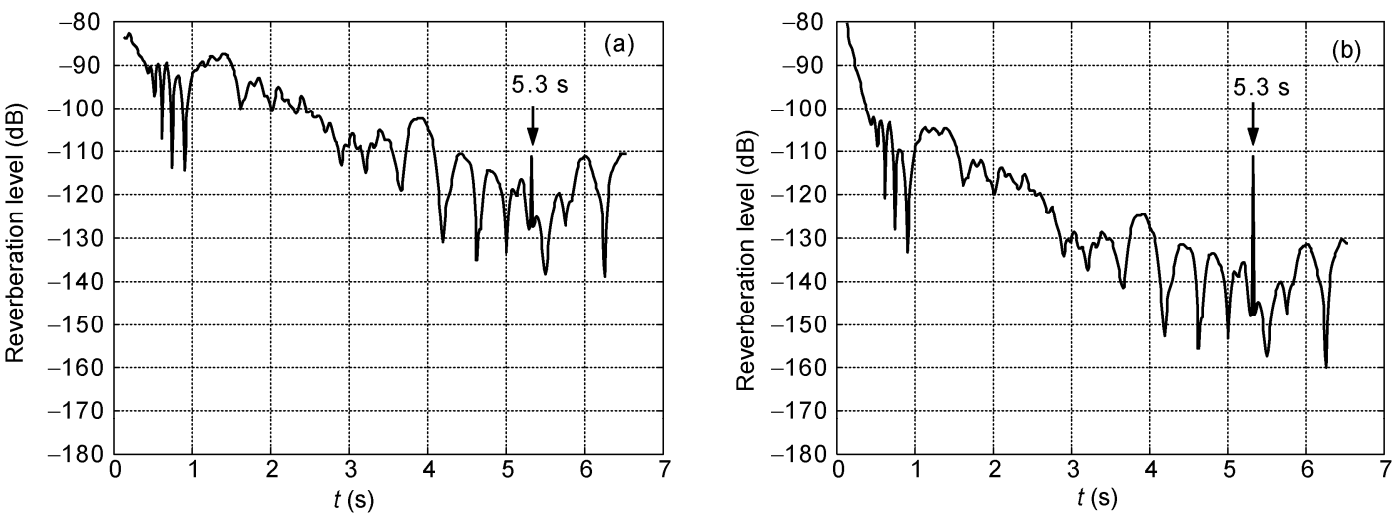

Figure 5 The resulting reverberation levels after passive reverberation nulling using the projection matrix given in eq. (13) (a), and passive reverberation nulling using the projection matrix given in eq. (15) (b), when $\Delta R=80 \mathrm{~m}$. 

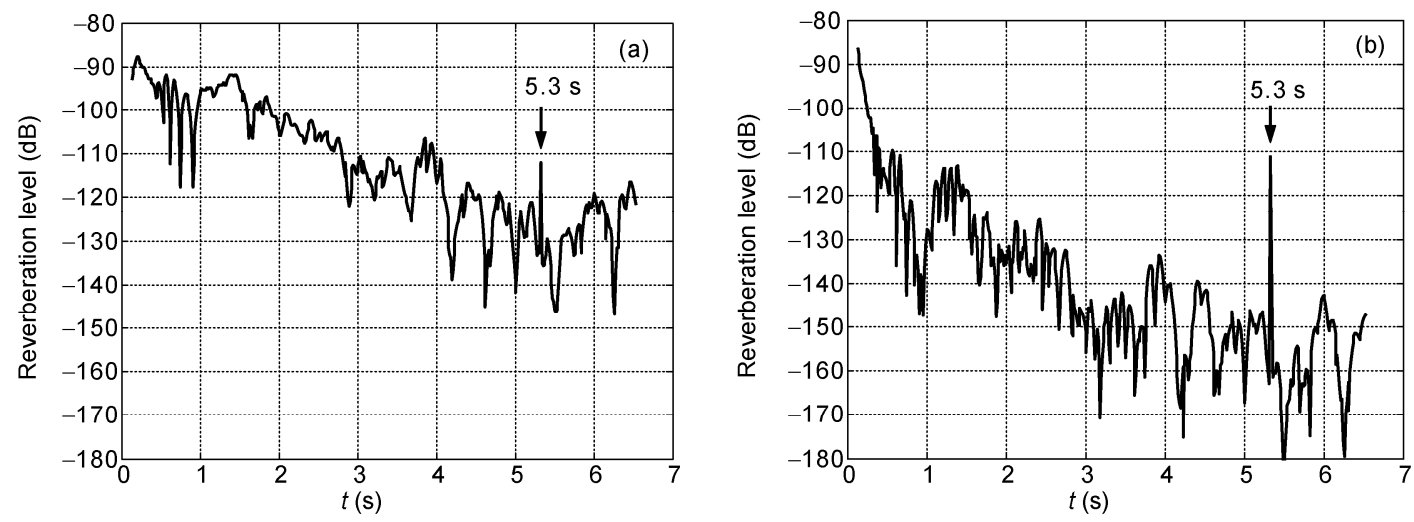

Figure 6 The resulting reverberation levels after passive reverberation nulling using optimal weighting vector based on direct forward prediction (a) and passive reverberation nulling using optimal weighting vector based on forward prediction using waveguide invariance (b) when $\Delta R=80 \mathrm{~m}$.

are lower, which is due to the normalization of optimal weight vector in eq. (16). Actually, the capability of reverberation nulling by optimal weight vector and project matrix is identical, while the former can further enhance the target echo, therefore obtaining higher echo-to-reverberation ratio.

The effect of $\Delta R$ increment on echo-to-reverberation ratio enhancement is examined. Figure 7 presents the echo-toreverberation ratio enhancement results by two passive reverberation nulling methods, where the optimal weighting vectors are obtained based on forwardinging prediction (denoted as RUFP) and the improved forwarding prediction using waveguide invariant (denoted as RUWI) proposed in this paper. The solid line at about $10 \mathrm{~dB}$ denotes the echoto-reverberation ratio of the original returns from BS transmission. As shown in the figure, if $\Delta R$ is small, both methods are effective for echo-to-reverberation ratio enhancement. Furthermore, when $\Delta \mathrm{R}$ increases, both of the methods become less effective for echo-to-reverberation enhancement. However, it is noticeable that the proposed method RUWI produced appreciably higher echo-to-reverberation

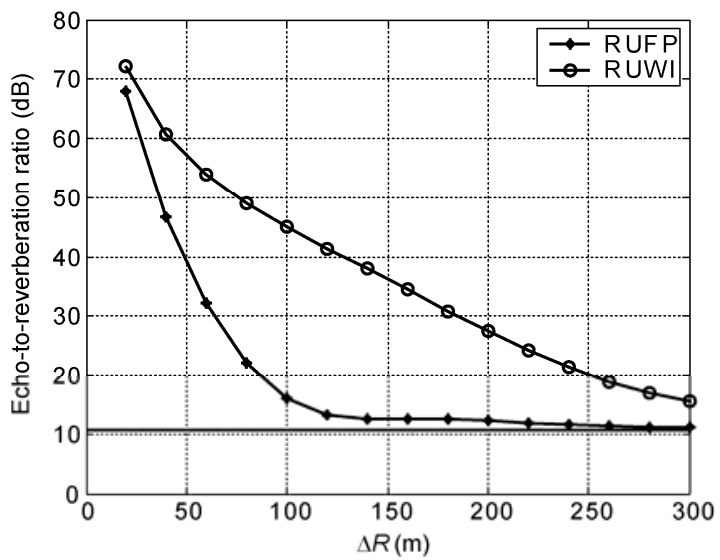

Figure 7 The echo-to-reverberation ratio enhancement results by two reverberation nulling methods, where the optimal weighting vectors are obtained respectively based on forwarding prediction (denoted as RUFP) and the improved forwarding prediction using waveguide invariant (denoted as RUWI), as $\Delta R$ increases. ratio at each $\Delta R$ case compared with the method RUFP.

\section{Conclusion}

In order to remove the limitations of current time reversal reverberation nulling method in practical use, a passive reverberation nulling and echo enhancement method using waveguide invariance is proposed in this paper. The basic theory of the method is that the reverberation subspace for the target range cell can be predicted from the reverberation returns scattered from a range cell located before the target using the waveguide invariance. Passive reverberation nulling using this predicted reverberation subspace can suppress the reverberation components in the target echo while the target echo is maintained. In addition, a range-depend optimal weight vector is deduced rather than a projector matrix to null the reverberation component meanwhile maximizing the target echo strength, therefore, enhancing the echo-to-reverberation ratio. The performance of the reverberation nulling and echo enhancement method proposed in this paper is studied by numerical simulations in typical shallow water environment. Conclusion is drawn as follows:

(1) Since passive processing is used, the proposed method eliminates the complexity of the active time reversal reverberation nulling, which requires active transmissions with different nulling excitation weighting vectors for all range cells potentially containing targets. Moreover, because a pure and exact reverberation subspace for the target range is obtained by forward predicting, this method successively overcomes the deficiencies of current passive reverberation nulling, such as simultaneous nulling of the target echo and the bottom reverberation.

(2) Although using optimal weighting vector of SRA performs almost equivalently as the case using the project matrix on suppression of the reverberation components, using optimal weighting vector can also maximize the target echo strength, ultimately resulting in much higher echo-toreverberation ratio. 
This work was supported by the National Natural Science Foundation of China (10974153) and Natural Science Foundation of Shaanxi Province (SJ08A08)

1 Fink M. Time-reversed acoustics. Sci Am, 1999, 281: 91-97

2 Kuperman W A, Hodgkiss W S, Song H C, et al. Phase conjugation in the ocean: Experimental demonstration of an acoustic time-reversal mirror. J Acoust Soc Am, 1998, 103: 25-40

3 Tanter M, Thomas J L, Fink M. Time reversal and the inverse filter. J Acoust Soc Am, 2000, 108: 223-234

4 Rouseff D, Jackson D R, Fox W L J, et al. Underwater acoustic communication by passive-phase conjugation: Theory and experimental results. IEEE J Oceanic Eng, 2001, 26: 821-831

5 Edelmann G, Song H C, Kim S, et al. Underwater acoustic communication using time reversal. IEEE J Oceanic Eng, 2005, 30: 852-864

6 Walker S C, Kuperman W A, Roux P. Active waveguide Green's function estimation with application to time-reversal focusing without a probe source in a range-independent waveguide. J Acoust Soc Am, 2006, 120: 2755-2763

7 Kim S, Kuperman W A, Hodgkiss W S, et al. Echo-to-reverberation enhancement using a time reversal mirror. J Acoust Soc Am, 2004, 115: 1125-1131

8 Song H C, Kim S, Hodgkiss W S, et al. Environmentally adaptive reverberation nulling using a time reversal mirror. J Acoust Soc Am, 2004, 116: 762-768

9 Song H C, Hodgkiss W S, Kuperman W A, et al. Experimental demonstration of adaptive reverberation nulling using time reversal. J Acoust Soc Am, 2005, 118: 1381-1387

10 Song H C, Hodgkiss W S, Kuperman W A, et al. Passive reverberation nulling for target enhancement. J Acoust Soc Am, 2007, 122: 3296-3303

11 Lingevitch J F, Song H C, Kuperman W A. Time reversed reverberation focusing in a waveguide. J Acoust Soc Am, 2002, 111: 2609-2614

12 Prada C, Manneville S, Spoliansky D, et al. Decomposition of the time reversal operator: Detection and selective focusing on two scatters. J Acoust Soc Am, 1996, 99: 2067-2076

13 Mordant N, Prada C, Fink M. Highly resolved detection and selective focusing in a waveguide using the D.O.R.T. method. J Acoust Soc Am, 1999, 105: 2634-2642

14 Gaumond C F, Fromm D M, Lingevitch J F, et al. Demonstration at sea of the decomposition-of-the-time-reversal-operator technique. J
Acoust Soc Am, 2006, 119: 976-990

15 Robert J L, Fink M. Green's function estimation in speckle using the decomposition of the time reversal operator: Application to aberration correction in medical imaging. J Acoust Soc Am, 2008, 123: 866-877

16 Prada C, Rosny J, Clorennec D, et al. Experimental detection and focusing in shallow water by decomposition of the time reversal operator. J Acoust Soc Am, 2007, 122: 761-768

17 Guo G Q, Yang Y X, Sun C. Echo-to-reverberation enhancement based on decomposition of time reversal operator with forward and backward reverberation nulling constrains. Chin J Acoust, 2009, 28: 38-50

18 Prada C, Thomas J L, Fink M. The iterative time reversal process: Analysis of the convergence. J Acoust Soc Am, 1995, 97: 62-71

19 Edelmann G F, Lingevithe J F, Gaumond C F, et al. Comparison of a subrank to a full-rank time-reversal operator in a dynamic ocean. J Acoust Soc Am, 2007, 122: 2706-2714

20 Brekhovskikh L M, Lysanov Y P. Fundamentals of Ocean Acoustics. 3rd ed. New York: Springer-Verlag Press, 1991. 143-148

21 Kuperman W A, D'Spain G L. Ocean acoustic interference phenomena and signal processing. AIP Conference Processings, 2002

22 Zhang R H, Su X X, Li F H. Improvement of low-frequency acoustic spatial correlation by frequency-shift compensation. Chin Phys Lett, 2006, 23: 1838-1841

23 Song H C, Kuperman W A, Hodgkiss W S. A time-reversal mirror with variable range focusing. J Acoust Soc Am, 1998, 103: 3234-3240

24 Hodgkiss W S, Song H C, Kuperman W A, et al. A long-range and variable focus phase-conjugation experiment in shallow water. $\mathrm{J}$ Acoust Soc Am, 1999, 105: 1597-1604

25 Carmillet V, Ambalard P O, Jourdain G. Detection of phase or frequency modulated signals in reverberation noise. J Acoust Soc Am, 1999, 105: 3375-3389

26 Brooks L A, Kidner M R F, Zander A C, et al. Techniques for extraction of the waveguide invariant from interference patterns in spectrograms. In: Proceedings of Acoustics 2006, Christchurch, New Zealand, 2006

27 Ellis D D. A shallow-water normal-mode reverberation model. J Acoust Soc Am, 1995, 97: 1804-1814

28 Kevin L. Bottom reverberation in shallow water: Coherent properties as a function of bandwidth, waveguide characteristics, and scatterer distributions. J Acoust Soc Am, 1999, 106: 3240-3254

29 Porter M B. The KRAKEN normal mode programs. SM-245, Undersea Research Centre, La Spezia, Italy, 1991

Open Access This article is distributed under the terms of the Creative Commons Attribution License which permits any use, distribution, and reproduction in any medium, provided the original author(s) and source are credited. 